\title{
BMJ Open Fear of cancer recurrence in peritoneal malignancy patients following complete cytoreductive surgery (CCRS) and hyperthermic intraperitoneal chemotherapy (HIPEC): an observational study protocol
}

Rayan Taher (D) , Sophia Stanford, Norman Carr, Nancy Vanderpuye, Kandiah Chandrakumaran

To cite: Taher R, Stanford S, Carr N, et al. Fear of cancer recurrence in peritoneal malignancy patients following complete cytoreductive surgery (CCRS) and hyperthermic intraperitoneal chemotherapy (HIPEC): an observational study protocol. BMJ Open 2022;12:e057294. doi:10.1136/ bmjopen-2021-057294

- Prepublication history for this paper is available online. To view these files, please visit the journal online (http://dx.doi. org/10.1136/bmjopen-2021057294).

Received 10 September 2021 Accepted 20 December 2021

Check for updates

(c) Author(s) (or their employer(s)) 2022. Re-use permitted under CC BY-NC. No commercial re-use. See rights and permissions. Published by BMJ.

Peritoneal Malignancy Institute, Hampshire Hospitals NHS Foundation Trust, Basingstoke, Hampshire, UK

Correspondence to

Rayan Taher;

rayan.taher@hhft.nhs.uk

\section{ABSTRACT}

Introduction Fear of cancer recurrence (FCR) is correlated with higher depression levels, worse quality of life and increased utilisation of healthcare services. There is no research on FCR in peritoneal malignancy (PM) patients-a rare type of abdominal cancer. This study aims to explore the prevalence, trajectory, demographic and clinical characteristics that are associated with FCR and its relationship with quality of life in PM patients.

Methods and analysis This is a cross-sectional study. Validated measures will be used to collect data on the levels of FCR (Fear of Cancer Recurrence InventoryShort Form) and quality of life (36-Item Short-Form Health Survey) of PM patients who have had surgery in the last 5 years at the Peritoneal Malignancy Institute in Basingstoke Hospital (minimum $\mathrm{N}=260$ ). Descriptive statistics, Pearson $\chi^{2}$ tests and correlational tests will be used to analyse the data.

Ethics and dissemination Ethical approval was obtained from the HRA and Health and Care Research Wales (HCRW). The results of this study will be shared with the participants of this study, presented at conferences and PM patients' days in the form of presentations or posters, and published in a scientific journal.

Discussion The results of this exploratory study will be used to inform a multicentre observational study to explore the effect of FCR on PM patients' mental health (depression and anxiety), quality of life and healthcare utilisation which will inform a multicentre randomised controlled trial to assess the effectiveness of using evidenced-based interventions to lower FCR in PM patients.

\section{INTRODUCTION}

Peritoneal malignancy $(\mathrm{PM})$ is a common clinical problem. It may be metastatic from gastrointestinal tract cancers or rarely may arise as a primary neoplasm of the peritoneum, usually malignant mesothelioma. ${ }^{12} \mathrm{At}$ the Peritoneal Malignancy Institute (PMI),
Strengths and limitations of this study

- This is the first study to assess levels of fear of cancer recurrence (FCR) and its relation to quality of life in peritoneal malignancy (PM) patients using a cross-sectional study design.

- This study will collect cross-sectional data from PM patients in five different groups depending on time since treatment (up to 5 years postsurgery) which will allow for a preliminary exploration of FCR over time post-treatment.

- The study uses self-report measures to measure FCR and quality of life which may pose a risk of cognitive bias and may result in a recruitment bias as those with high FCR are more likely to decline to avoid the topic.

Basingstoke the main types of PM treated are pseudomyxoma peritonei (PMP), colorectal peritoneal metastases (CPM) and peritoneal mesothelioma. PMP is a syndrome of mucinous intra-abdominal neoplasia that usually arises from a primary mucinous tumour of the appendix. ${ }^{3}{ }^{4}$ Colorectal cancer is a common primary site of PM. ${ }^{5}{ }^{6}$ Histologically it is generally adenocarcinoma and may be mucinous or non-mucinous. ${ }^{5}{ }^{6}$ Primary peritoneal malignant mesothelioma is rare and is associated with asbestos exposure. ${ }^{7}$ Selected PM patients undergo a combination of cytoreductive surgery (CRS) and hyperthermic intraperitoneal chemotherapy (HIPEC). ${ }^{8}$ The outcome of the surgery is considered complete if all PM nodules larger than $2.5 \mathrm{~mm}$ are removed. ${ }^{8}$ Unfortunately, studies have shown that PM can recur even after having complete CRS. ${ }^{9}$ Still, there is 
no study on the fear of cancer recurrence (FCR) in PM patients.

FCR is defined as 'fear or worry that cancer will return, progress, or metastasis'. ${ }^{10}$ Patients with cancer report FCR as their number one unmet need. ${ }^{11}{ }^{12}$ It is harder to adjust to than the initial diagnosis. ${ }^{13}$ Patients with higher FCR report lower quality of life up to 6 years post-treatment, ${ }^{10}$ worsened relationships, difficulties at work and mental health problems such as anxiety, post-traumatic stress and depression. ${ }^{10}$ Even though FCR is a common reaction to cancer, it can become chronic and disabling in up to $70 \%$ of patients. ${ }^{10}$ A study on FCR among patients with colorectal cancer found that $4 \%-85 \%$ of them struggle with FCR, and $38 \%$ struggle with clinical/high FCR. ${ }^{14} \mathrm{~A}$ study on FCR in patients with breast cancer found that $70 \%$ of their participants (N=218) struggled with clinical/ high FCR. ${ }^{11}$ FCR does not subside with time.${ }^{10}{ }^{15}$ High FCR levels are correlated with an increase in healthcare cost because patients with high FCR are more likely to seek medical services for reassurance or may avoid follow-up out of fear and thus requiring more invasive treatments that could be avoided. ${ }^{11}$ A survey with oncology health professionals found that $31 \%$ of doctors spent more than a quarter of their follow-up time addressing FCR concerns. ${ }^{11}$

Evidence shows that uncertainty is positively correlated with FCR levels. ${ }^{15}$ A qualitative study that explored the experiences of PM patients found 'uncertainty' to be one of the main themes reported by patients. ${ }^{16}$ We hypothesise that the rare nature of the disease and the uncertainty around it increases PM patients' risk of developing FCR. PM patients in the UK report relying solely on the few PM specialists available (two centres in the UK) and online groups for support because of the limited knowledge available around PM in other services. ${ }^{16}$ For PM patients, high levels of FCR combined with limited resources to seek support might result in an increase in pressure and demand on already strained services. For those reasons, along with the negative impact of FCR on patients' mental health and quality of life, it is important to address FCR in PM patients. This study aims to assess the prevalence and trajectory of FCR, and its relationship to a number of demographic and clinical characteristics and quality of life.

\section{Aim}

The primary outcome of this study is:

- Assess the severity levels of FCR in PM patients who have had surgery in the past 5 years, its trajectory over time, and its relationship with quality of life in PM patients postsurgery.

The secondary outcomes of this study are:

- Assess the characteristics that affect FCR in PM patients (age, gender, income, education, ethnicity, relationship status, whether they have any dependents, disease type (PMP, CPM or peritoneal mesothelioma), time since treatment (complete cytoreductive surgery (CCRS) and HIPEC), disease grade, mental health history and mental health history at time of surgery (diagnosis and prescriptions of psychotropic medications).

- Inform a multicentre observational study to explore the effect of FCR on PM patients' mental health (depression and anxiety), quality of life and healthcare utilisation to inform a multicentre randomised controlled trial to assess the effectiveness of using evidenced-based interventions to lower FCR in PM patients.

\section{METHODS}

\section{Study design}

This is an exploratory cross-sectional study.

\section{Patient and public involvement}

This protocol was shared with the patient and public involvement (PPI) group during its development to ensure the engagement of PM patients in the design process of this study. The protocol was edited based on the feedback from PM patients in the PPI group.

\section{Participants}

PM patients who had a complete CRS and HIPEC (surgery) in the last 5 years at the PMI in Basingstoke Hospital will be invited to take part in this study.

\section{Inclusion and exclusion criteria}

Inclusion criteria: PM patients who had a complete CRS and HIPEC (surgery) in the last 5 years at the PMI in Basingstoke Hospital. Exclusion criteria: below the age of 18, disease recurrence and any cognitive or language difficulties that may affect results. Participants who have also had other malignancies that they were treated for (cancer free at the time of the study) will not be excluded from this study.

\section{Material}

- Fear of Cancer Recurrence Inventory-Short Form (FCRI-SF) will be used to measure FCR. The FCRI-SF is a 9 item Likert scale that was derived from the 42-item FCRI. ${ }^{17}$ It is used to measure FCR by assessing the presence and severity of FCR related intrusive thoughts in patients with cancer. ${ }^{17}$ Scores range from 0 to 36 , with higher scores indicating higher levels of FCR. ${ }^{17}$ Thirteen is the scale's suggested cut-off point as it has shown optimal sensitivity (88\%) and specificity (75\%) compared with the other previously used cut-off points $(>16$ and $>22)$ in screening for clinical/severe FCRwhich is associated with impaired physical, emotional, cognitive and social functioning. ${ }^{18} 19$ The FCRI-SF has a strong internal consistency $(\alpha=0.95)$, temporal stability $(\mathrm{r}=0.89)$ and construct validity. ${ }^{20}$

- The 36-Item Short-Form Health Survey (SF-36) will be used to assess participants' quality of life. The SF-36 is a self-administered health survey that is used to assess patients' quality of life. ${ }^{21}$ The SF-36 contains eight domains: physical functioning (10 items), rolephysical (4 items), bodily pain (2 items), general 
health (5 items), vitality (4 items), social functioning (2 items), role-emotional (3 items) and mental health (5 items). ${ }^{22}$ All items relate to the 4 weeks preceding the questionnaire's completion. ${ }^{23}$ The SF-36 has two component summary scores: (1) Physical Component Summary which encompasses physical function, role physical and bodily pain, and (2) Mental Component Summary which encompasses social function, role emotional and mental health. ${ }^{23}$ Total scores range from 0 to 100 with higher scores indicating a better quality of life. $^{21}$

- Clinical and demographic characteristics will be collected to assess the relationship between them and FCR. Participants will be asked about their age, gender, income, education, ethnicity, relationship status, whether they have any dependents, disease type (PMP, CPM or peritoneal mesothelioma), time since treatment (CCRS and HIPEC), disease grade, mental health history and mental health history at the time of surgery (diagnosis and prescriptions of psychotropic medications).

\section{Procedure}

The research and data coordinator will generate a report of all the patients who have had a complete CRS and HIPEC in the last 5 years at the PMI in Basingstoke Hospital, and meet the inclusion and exclusion criteria from the hospital's database. The generated report will be ordered based on patients' surgery date and will be divided into five lists as such:

\begin{tabular}{lllll}
\hline List 1 & List 2 & List 3 & List 4 & List 5 \\
\hline Patients who & Patients who Patients & Patients who & Patients who \\
had CCRS & had CCRS & who had & had CCRS & had CCRS \\
and HIPEC & and HIPEC in CCRS and & and HIPEC in & and HIPEC in \\
in the last & the last 1-2 & HIPEC in & the last 3-4 & the last 4-5 \\
0-1 year & years & $\begin{array}{l}\text { the last 2-3 } \\
\text { years }\end{array}$ & years & years \\
& & & & \\
\hline
\end{tabular}

The first 52 patients of every list will be invited to participate in this study. This will be done to ensure that participants' sample sizes are comparable over time to investigate the trajectory of FCR in the 5 years posttreatment. A copy of the participant information sheet along with a letter that explains the reason why these patients are receiving this invitation will be mailed to all potential participants. The letter will also inform participants that they will receive a call within 1-2 weeks' time from the research assistant at the PMI in Basingstoke Hospital to answer any questions they have and ask for their consent to participate. The letter will also include an email and a phone number to contact if they would prefer not to be contacted about this study. On the day of the call, the research assistant will explain the study and what it involves, answer any questions that potential participants have, and ask if they want to participate in the study (verbal consent). Participants who consent to participate will be asked whether they would like to receive the questionnaires via mail or email. Participants who choose mail will be sent an envelope that contains the participant information sheet, FCRI-SF, SF-36, demographic and clinical questions, and a prepaid return envelope. Those patients will be asked to kindly return the completed questionnaires within 2 weeks from receiving them. The research assistant will call these patients after a week of sending the questionnaires to ensure that they have received them, ask if they have any questions, and remind them to send the completed questionnaires within 2 weeks from receiving them. Participants who choose email will be emailed a link using Microsoft Forms (per the Trust's data security regulations) that contains the participant information sheet, FCRI-SF, SF-36, and the demographic and clinical questions, and asked to complete the questionnaires within 2 weeks of receiving them. Participants who receive the questionnaires by email will be sent an email 1 week later to ask if they have any questions and remind them to send the completed questionnaires in the following week. Even though it may be more accurate to collect the clinical data such as disease type and grade from the hospital's database rather than the patients themselves, for the purposes of the study (measuring FCR) it is more important what participants know/believe about their disease that is, if a patient has a high-grade disease but is unaware of it that will not affect their 'fear' of cancer recurrence. All collected data will be stored on an encrypted excel spreadsheet on a National Health Service (NHS) computer and in a folder in the PMI Research room at Hampshire Hospitals NHS Foundation Trust in a locked metal cabinet only accessible by the study team.

We are currently in the process of recruiting and collecting data for this study. Recruitment and data collection for this study started on 4 October 2021. We estimate that it will take 2-3 months to complete the data collection process and 3-4 months to analyse the data, write the paper and submit it for publication. In total, we expect this study to take up to 7 months to complete.

\section{Sample size and statistics}

To the authors' knowledge, there is no previous study on FCR among PM patients, hence, there is a known effect size to be used to calculate the sample size. For this reason, a standardised Cohen's $\mathrm{d}=0.25$ was used to detect a medium to large effect size. ${ }^{24}$

To detect an effect $|p|$ of 0.25 (Cohen's d) with $80 \%$ power in a one-way between subjects Analysis of Varaince (ANOVA) $(\alpha=0.05)$, the required sample is 52 participants in each group (total=260). Sample size was calculated with R V.3.6.3 program (R Foundation for Statistical Computing, Vienna, Austria).

Categorical variable will be outlined with frequencies and percentages and continuous data with mean and SD. Analysis of categorical data will be performed using the Pearson $\chi^{2}$ test or Fisher's exact test where appropriate. A $p$ value of less than 0.05 is considered statistically significant. A line graph using group mean scores will be used to plot the trajectory of FCR and quality of life over time. 
Correlation tests will be conducted to explore the relationship between FCR and quality of life. Data will be analysed using SPSS V.26 (IBM).

\section{ETHICS AND DISSEMINATION}

Ethical approval was obtained from the HRA and Health and Care Research Wales (HCRW) (IRAS ID: 295431). Below are the main ethical considerations of this study:

- Participants with language and/or cognitive difficulties who are unable to complete the questionnaires without assistance will be excluded from this study. This will be done because PM patients are dispersed all over the UK as it is a specialist service. Thus, it will be logistically difficult to arrange for translators to support these participants. We are also aware that participants might be uncomfortable answering these questions (sensitive topic) in front of translators, which might cause participants discomfort and affect results.

- Participants will be given the option to choose whether they would like to receive the questionnaires by mail or email. This is done to accommodate all age groups and different levels of technical skills.

- All data (mail and online) will not include any identifying information, to protect patients' confidentiality.

- Participants will be informed in the participant information sheet that the questions will address their thoughts around the disease and its recurrence, and their quality of life both mental and physical, and that these questions might cause some distress. Participants will also be informed that they can withdraw from the study at any point and that that will not affect their rights or any future treatment. They will also be encouraged to contact the research assistant if completing the questionnaires causes them any distress. If they do so, the research assistant-in discussion with them and with their permission-will refer them to their local General Practitioner and/or other emotional support services in their area.

- All data and information collected during this study will be considered and treated as confidential by the study team.

- The results of this study will be shared with the study's participants, presented at conferences and PM patients' days in the form of presentations or posters, and published in a scientific journal.

\section{DISCUSSION}

According to Butow et al: 'FCR is a critical target for optimal survivorship care' ${ }^{25}$ We hypothesise that the limited knowledge and uncertainty around PM increases the risk of FCR in this population. This study will explore the prevalence, trajectory and potential risk factors of FCR in PM patients and its relationship to quality of life. The results of this study will inform a multicentre study to explore the effect of FCR on PM patients' mental health (depression and anxiety), quality of life and healthcare utilisation which will inform a multicentre randomised controlled trial to assess the effectiveness of using evidenced-based interventions to lower FCR in PM patients.

One of the limitations of this study is that it is a crosssectional study; thus, no causal relationships can be drawn from the results. Another limitation is the use of self-report measures to collect FCR and quality of life data increases the risk of cognitive bias and recollection distortion which might affect results. There might also be a selection bias in the recruitment process as those who suffer from FCR are more likely to avoid participating in a study about FCR. Even though all participants will be cancer free-to the best of their knowledge-at the time of the study (inclusion criteria), some participants might have had other malignancies in the past. Participants will be asked to focus on their PM experience when answering the questions however that might still affect results and pose a limitation for this study. This study is assessing the trajectory of FCR over time using a cross-sectional design (comparing different patients over time) thus individual differences may affect results (confounding variables).

The results of this exploratory cross-sectional study will be used to inform a multicentre study to explore the effect of FCR on PM patients' mental health (depression and anxiety), quality of life and healthcare utilisation. The results of both studies will then be used to conduct a multicentre randomised controlled trial to assess the effectiveness of using evidenced-based psychological interventions with high-risk patients on decreasing FCR levels and improving PM patients' quality of life.

The measure used is a self-report measure that poses risks of cognitive bias and recollection distortion

Twitter Sophia Stanford @SophiaStanford1

Acknowledgements We thank our colleagues at the Peritoneal Malignancy Institute at Basingstoke Hospital for their support in planning this study.

Contributors RT and SS designed the study and developed the protocol. NC is a pathologist and helped with medical background included in the study. NV is a psychologist and helped review the study from a mental health perspective. $\mathrm{KC}$ is a statistician and helped calculate the sample size and proposed statistics for the study.

Funding This work was supported by the Peritoneal Malignancy Institute charitable fund. As the study has been written by an employee of, and is sponsored by the NHS, NHS liability insurance will apply.

Competing interests None declared.

Patient and public involvement Patients and/or the public were involved in the design, or conduct, or reporting, or dissemination plans of this research. Refer to the Methods section for further details.

Patient consent for publication Consent obtained directly from patient(s). Provenance and peer review Not commissioned; externally peer reviewed.

Open access This is an open access article distributed in accordance with the Creative Commons Attribution Non Commercial (CC BY-NC 4.0) license, which permits others to distribute, remix, adapt, build upon this work noncommercially, and license their derivative works on different terms, provided the original work is properly cited, appropriate credit is given, any changes made indicated, and the use is non-commercial. See: http://creativecommons.org/ licenses/by-nc/4.0/. 
ORCID iD

Rayan Taher http://orcid.org/0000-0001-8592-527X

\section{REFERENCES}

1 Carr NJ. New insights in the pathology of peritoneal surface malignancy. J Gastrointest Oncol 2021;12:S216-29.

2 Kyang LS, Alzahrani NA, Valle SJ, et al. Long-term survival outcomes of cytoreductive surgery and perioperative intraperitoneal chemotherapy: single-institutional experience with 1225 cases. J Surg Oncol 2019;120:794-802.

3 Ahmadi N, Kostadinov D, Sakata S, et al. Managing recurrent pseudomyxoma peritonei in 430 patients after complete cytoreduction and HIPEC: a dilemma for patients and surgeons. Ann Surg Oncol 2021;28:7809-20.

4 Huang Y, Alzahrani NA, Chua TC, et al. Histological subtype remains a significant prognostic factor for survival outcomes in patients with appendiceal mucinous neoplasm with peritoneal dissemination. Dis Colon Rectum 2017;60:360-7.

5 März L, Piso P. Treatment of peritoneal metastases from colorectal cancer. Gastroenterol Rep 2015;3:gov044-302.

6 Sánchez-Hidalgo JM, Rodríguez-Ortiz L, Arjona-Sánchez Álvaro, et al. Colorectal peritoneal metastases: optimal management review. World J Gastroenterol 2019;25:3484-502.

7 Consonni D, Calvi C, De Matteis S, et al. Peritoneal mesothelioma and asbestos exposure: a population-based case-control study in Lombardy, Italy. Occup Environ Med 2019;76:545-53.

8 Chiu C-C, Lee P-H, Chang C-S, et al. Correspondence to 'Treatment of PERItoneal disease in Stomach Cancer with cytOreductive surgery and hyperthermic intraPEritoneal chemotherapy: PERISCOPE I initial results'. Br J Surg 2021;108:e93.

9 Zhu Y, Hanna N, Boutros C, Cherif Boutros Jr H, et al. Assessment of clinical benefit and quality of life in patients undergoing cytoreduction and hyperthermic intraperitoneal chemotherapy (HIPEC) for management of peritoneal metastases. J Gastrointest Oncol 2013;4:62.

10 Crist JV, Grunfeld EA. Factors reported to influence fear of recurrence in cancer patients: a systematic review. Psychooncology 2013;22:978-86.

11 Butow PN, Bell ML, Smith AB, et al. Conquer fear: protocol of a randomised controlled trial of a psychological intervention to reduce fear of cancer recurrence. BMC Cancer 2013;13:1-10.
12 Simard S, Thewes B, Humphris G, et al. Fear of cancer recurrence in adult cancer survivors: a systematic review of quantitative studies. $J$ Cancer Surviv 2013;7:300-22.

13 Fardell JE, Thewes B, Turner J, et al. Fear of cancer recurrence: a theoretical review and novel cognitive processing formulation. $J$ Cancer Surviv 2016;10:663-73.

14 Custers JAE, Gielissen MFM, Janssen SHV, et al. Fear of cancer recurrence in colorectal cancer survivors. Support Care Cancer 2016;24:555-62.

15 Butow PN, Fardell JE, Smith A. August). fear of cancer recurrence: an overview and Australian perspective. in cancer forum. Australia: The Cancer Council, 2015: 39. P 95.

16 Witham G, Willard C, Ryan-Woolly B, et al. A study to explore the patient's experience of peritoneal surface malignancies: pseudomyxoma peritonei. Eur J Oncol Nurs 2008;12:112-9.

17 Peng L, Huang W, Zhang W, et al. Psychometric properties of the short form of the fear of cancer recurrence inventory (FCRI) in Chinese breast cancer survivors. Front Psychiatry 2019;10:537.

18 Simard S, Savard J. Screening and comorbidity of clinical levels of fear of cancer recurrence. J Cancer Surviv 2015;9:481-91.

19 Smith AB, Costa D, Galica J, et al. Spotlight on the fear of cancer recurrence inventory (FCRI). Psychol Res Behav Manag 2020;13:1257-68.

20 Lynch FA, Katona L, Jefford M, et al. Feasibility and Acceptability of Fear-Less: A Stepped-Care Program to Manage Fear of Cancer Recurrence in People with Metastatic Melanoma. J Clin Med 2020;9:2969.

21 Silva GE, Goodwin JL, Vana KD, et al. Obstructive sleep apnea and quality of life: comparison of the SAQLI, FOSQ, and SF-36 questionnaires. Southwest J Pulm Crit Care 2016;13:137-49.

22 Finkelstein FO, van Nooten F, Wiklund I, et al. Measurement properties of the Short Form-36 (SF-36) and the Functional Assessment of Cancer Therapy - Anemia (FACT-An) in patients with anemia associated with chronic kidney disease. Health Qual Life Outcomes 2018;16:111.

23 Hoffner M, Bagheri S, Hansson E, et al. Sf-36 shows increased quality of life following complete reduction of postmastectomy lymphedema with liposuction. Lymphat Res Biol 2017;15:87-98.

24 Cohen J. Statistical power analysis for the behavioral sciences. Academic press, 2013.

25 Butow P, Sharpe L, Thewes B, et al. Fear of cancer recurrence: a practical guide for clinicians. Oncology 2018;32:32-8. 